\title{
12-Week Effectiveness and Safety of Low-Density Lipoprotein Cholesterol-Lowering Therapy by Proprotein Convertase Subtilisin/Kexin Type 9 Inhibition in Patients With Familial Hypercholesterolemia and Hypercholesterolemia \\ - Data From a Real-World Observational Study of Evolocumab in Japan -
}

\author{
Koutaro Yokote, MD, PhD; Junya Ako, MD, PhD; Kazuo Kitagawa, MD, PhD; \\ Hyoe Inomata, MD, PhD; Toshihiko Sugioka; Keiko Asao, MD, PhD; \\ Yasuhiko Shinmura; Junichiro Shimauchi; Tamio Teramoto, MD, PhD
}

\begin{abstract}
Background: Evolocumab is the first monoclonal antibody against proprotein convertase subtilisin/kexin type 9 (PCSK9) approved in Japan for the treatment of patients with familial hypercholesterolemia $(\mathrm{FH})$ and hypercholesterolemia $(\mathrm{HC})$. This study assessed the 12-week effectiveness and safety of low-density lipoprotein cholesterol (LDL-C)-lowering therapy by PCSK9 inhibition in patients with $\mathrm{FH}$ (homozygous $[\mathrm{HoFH}]$ or heterozygous $[\mathrm{HeFH}]$ ) and $\mathrm{HC}$ by analyzing evolocumab data collected in the real-world setting in Japan.
\end{abstract}

Methods and Results: Overall, 427 patients (mean \pm SD age, 61.6 \pm 13.8 years; female, 38.4\%; $28 \mathrm{HoFH}, 320 \mathrm{HeFH}, 79 \mathrm{HC}$ ), enrolled from 299 clinical sites, were included in the safety analysis set. The major cardiovascular risk factors were coronary artery disease $(77.3 \%)$, diabetes mellitus/impaired glucose tolerance (38.6\%), and hypertension (65.1\%). Median follow-up duration was 85.0 days. After 12 weeks of evolocumab treatment, the mean \pm SD percent change from baseline in LDL-C was $-45.5 \% \pm 27.0 \%$ $(n=23)$ in HoFH $(P<0.001$ vs. baseline; $t$-test), $-54.2 \% \pm 29.0 \%(n=280)$ in $\mathrm{HeFH}(P<0.001)$, and $-64.6 \% \pm 22.4 \%(n=72)$ in $\mathrm{HC}$ $(P<0.001)$ patients. The incidence of adverse drug reactions was $5.4 \%(23 / 427)$.

Conclusions: Results suggest that patients receiving evolocumab treatment in the real-world setting were predominantly those with $\mathrm{FH}$ and $\mathrm{HC}$ in the secondary prevention group. LDL-C-lowering effectiveness with evolocumab was observed in FH (both HoFH and $\mathrm{HeFH}$ ) and $\mathrm{HC}$ patients.

Key Words: Evolocumab; Familial hypercholesterolemia; Heterozygous; Homozygous; PCSK9 inhibition

$\mathbf{S}$ everal prior studies, both randomized and observational, have demonstrated that reduction in lowdensity lipoprotein cholesterol (LDL-C) levels in patients with hypercholesterolemia (HC), including those with familial hypercholesterolemia $(\mathrm{FH})$, by statin therapy has a beneficial effect on lowering the risk of cardiovascular (CV) events and CV death.,2 Thus, LDL-C is a key modifiable risk factor in the development of $\mathrm{CV}$ events. ${ }^{3}$

Evolocumab is the first approved monoclonal antibody against proprotein convertase subtilisin/kexin type 9(PCSK9) for clinical use in $\mathrm{FH}$ and $\mathrm{HC}$ in Japan. ${ }^{4}$ The results of the
FOURIER trial support the efficacy of evolocumab in combination with statin therapy in significantly reducing the risk of $\mathrm{CV}$ events (evaluated by major composite CV events) while reducing LDL-C by $59 \%$ compared with placebo. ${ }^{5}$ Recent clinical evidence-based guidelines, including those from the Japan Atherosclerosis Society (JAS) for FH and $\mathrm{HC}$, recommend maintaining LDL-C values $<70 \mathrm{mg} / \mathrm{dL}$, especially in patients at high risk for $\mathrm{CV}$ events. ${ }^{6}$

The Japanese package insert for evolocumab included randomized trials that provided efficacy and safety data for 565 Japanese subjects. ${ }^{4}$ Given that patients with FH, espe-

Received March 22, 2019; revised manuscript received April 11, 2019; accepted April 12, 2019; J-STAGE Advance Publication released online May 8, 2019 Time for primary review: 1 day

Department of Endocrinology, Hematology, and Gerontology, Chiba University Graduate School of Medicine, Chiba (K.Y.); Department of Cardiovascular Medicine, Kitasato University School of Medicine, Sagamihara (J.A.); Department of Neurology, Tokyo Women's Medical University School of Medicine, Tokyo (K.K.); Amgen Astellas BioPharma K.K., Tokyo (H.I., T.S., K.A., Y.S., J.S.); and Teikyo Academic Research Center, Teikyo University, Tokyo (T.T.), Japan

Mailing address: Koutaro Yokote, MD, PhD, Department of Endocrinology, Hematology, and Gerontology, Chiba University Graduate School of Medicine, 1-8-1 Inohana, Chuo-ku, Chiba 260-8670, Japan. E-mail: kyokote@faculty.chiba-u.jp

ISSN-2434-0790 All rights are reserved to the Japanese Circulation Society. For permissions, please e-mail: cr@j-circ.or.jp 


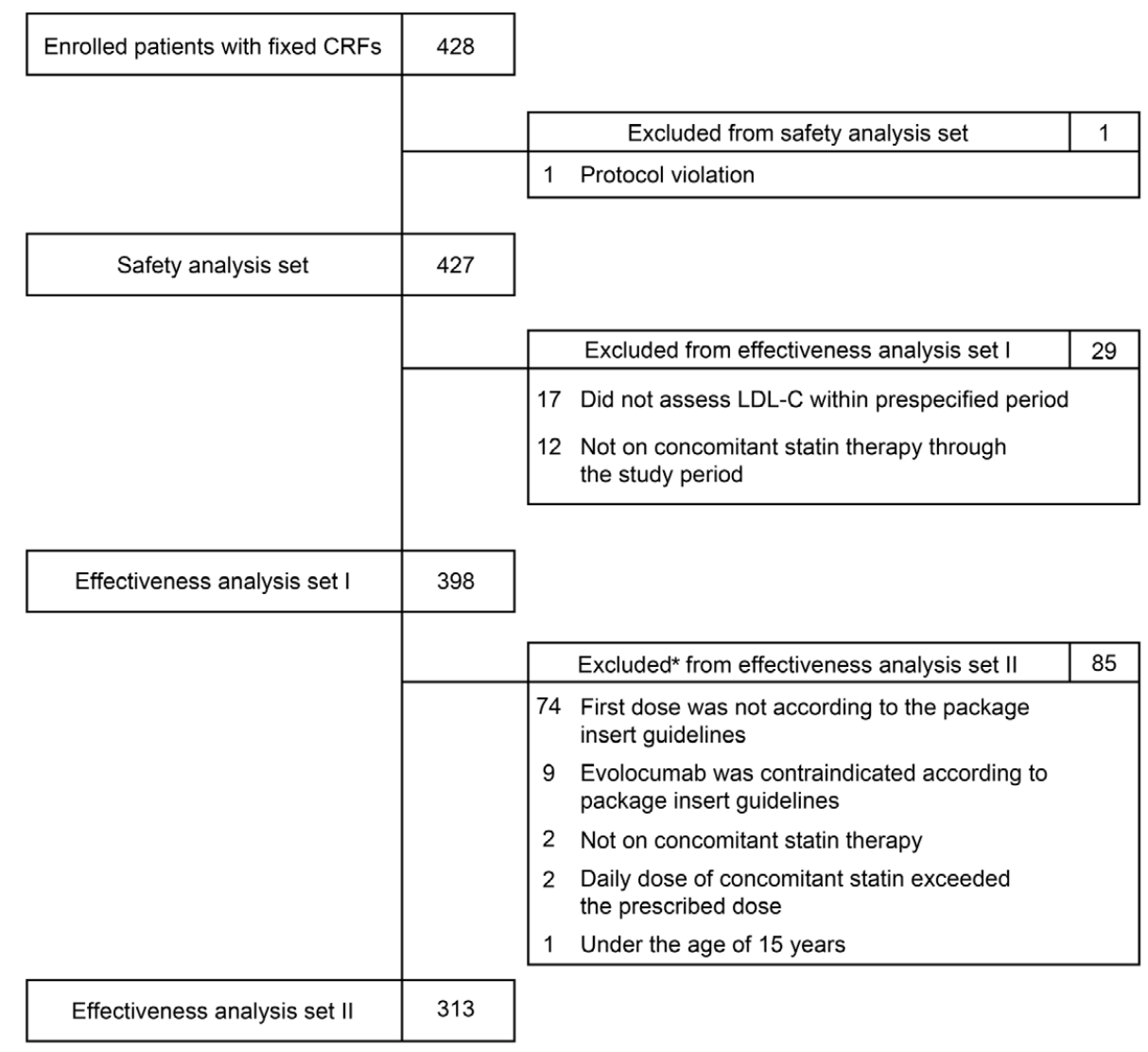

Figure 1. Patient Disposition. *Multiple reasons for exclusion were possible in a single case. CRF, case report form; LDL-C, low-density lipoprotein cholesterol.

cially those with homozygous $\mathrm{FH}(\mathrm{HoFH})$ are limited due to the rarity of this condition, only $7 \mathrm{HoFH}$ patients could be assessed in the clinical studies in Japan. ${ }^{7}$ The present post-marketing observational study provides descriptive data from the real-world use of evolocumab in Japanese patients to supplement the data in the Japan-new drug application (J-NDA). We investigated (1) the effectiveness of evolocumab, when used in combination with statins or other lipid medications, in lowering LDL-C levels in patients with $\mathrm{HC}$, specifically in those with $\mathrm{FH}$; (2) baseline demographics and characteristics of $\mathrm{FH}$ patients who were receiving evolocumab treatment; and (3) the safety profile of evolocumab in the real-world setting. Here, we present the safety and effectiveness from this ongoing observational study, in order to publish the information on evolocumab in the real-world setting in Japan in a timely manner.

\section{Methods}

\section{Study Design and Patients}

This was an observational, post-marketing surveillance (PMS) study conducted at 299 participating centers in Japan from 24 June 2016 (first subjects in) to 17 January 2018 (inclusive). The study population consisted of patients who were evolocumab naïve and who were prescribed evolocumab according to the approved Japanese package insert (i.e., those with $\mathrm{FH}$ or $\mathrm{HC}$ who were at high $\mathrm{CV}$ risk and who had inadequate response to statins). ${ }^{4}$ After the first subcutaneous injection of evolocumab, patients were monitored during routine clinic visits for 12 weeks. At each visit, data for adverse events (AE) and medication use were documented following patient interview. Evolocumab was prescribed according to the dosage guidelines in the package insert. In the case of inadequate response to statins, patients with $\mathrm{HC}$ (including heterozygous $\mathrm{FH}[\mathrm{HeFH}]$ ) received evolocumab $140 \mathrm{mg}$ every 2 weeks or $420 \mathrm{mg}$ once monthly, and those with $\mathrm{HoFH}$ received evolocumab $420 \mathrm{mg}$ once monthly or $420 \mathrm{mg}$ every 2 weeks.

$\mathrm{FH}$ and $\mathrm{HC}$ should be diagnosed as per the criteria specified in the 2012 and 2017 JAS guidelines. ${ }^{6.8}$ In the real-world clinical setting, however, physicians at the participating site could diagnose $\mathrm{FH}$ based on the clinical manifestations of $\mathrm{FH}$, such as xanthoma tuberosum and the presence of persistently elevated LDL-C levels, without performing a genetic test. Thus, it is possible that clinically diagnosed HoFH patients in the present study were potentially $\mathrm{HeFH}$ patients, given that the diagnoses were not confirmed on genetic testing.

Patients eligible for safety analyses had at least 1 documented visit after the index date (date of initiation of evolocumab). Patients in whom follow-up LDL-C data were available and who received concomitant statin were included in the effectiveness analysis set I; patients who were treated with the on-label dose regimen of evolocumab 
and statins were included in the effectiveness (sensitivity) analysis set II. To enable assessment of the long-term effectiveness and safety of evolocumab, this study will continue to collect data from 3,000 patients through a 2-year observation period.

The study protocol was approved by the institutional or ethics review board at each participating site or by a central ethical review board (Public Health Research Foundation, Tokyo). This study was conducted in accordance with the Pharmaceutical and Medical Devices Law, Good PostMarketing Study Practice in Japan, and the Declaration of Helsinki. The study sponsors take responsibility for the accuracy and completeness of the data and analyses. This study is registered at ClinicalTrials.gov (identifier, NCT02808403). Written informed consent was provided by all enrolled patients.

\section{Background Data}

All patient data were collected in a patient registration form or a case report form (CRF) using the Electronic Data Capture (EDC) system (PostMaNet, Fujitsu, Tokyo). The following data were collected at baseline: sex, age, body mass index, inpatient/outpatient setting at the time of initiation of evolocumab, diagnosis $(\mathrm{FH}[\mathrm{HeFH} / \mathrm{HoFH}] /$ $\mathrm{HC}$ ); FH-related findings including genetic tests for $\mathrm{FH}$ (whether conducted or not), tendon xanthoma, xanthoma tuberosum; past medical history including that of coronary artery disease (CAD), diabetes mellitus (DM)/impaired glucose intolerance (IGT), chronic kidney disease (CKD), non-cardiogenic cerebral infarction, peripheral artery disease (PAD), hypertension, low high-density lipoprotein cholesterol (HDL-C; defined as $<40 \mathrm{mg} / \mathrm{dL}$ ), smoking history, family history of premature CAD, risk category (according to JAS guidelines) ${ }^{6}$ using risk factor count, high-intensity statin use, ${ }^{9}$ concomitant lipid-lowering medications, prior medications, and prior LDL-C apheresis therapy. The LDL-C goal established according to the comprehensive JAS risk management goals ${ }^{6}$ was $<70 \mathrm{mg} / \mathrm{dL}$ in $\mathrm{FH}$ and $<100 \mathrm{mg} / \mathrm{dL}$ in $\mathrm{HC}$ patients for secondary prevention; and $<120 \mathrm{mg} / \mathrm{dL},<140 \mathrm{mg} / \mathrm{dL}$, and $<160 \mathrm{mg} / \mathrm{dL}$ in high-risk, moderate-risk, and low-risk categories, respectively, for primary prevention. The name of the statin, its daily dose, and treatment start and end dates were collected on the CRF. High-intensity statin use was defined as atorvastatin $\geq 10 \mathrm{mg}$ once daily (QD), rosuvastatin $\geq 5 \mathrm{mg}$ $\mathrm{QD}$, simvastatin $\geq 20 \mathrm{mg}$ QD, fluvastatin $\geq 80 \mathrm{mg}$ QD, pitavastatin $\geq 2 \mathrm{mg}$ QD, pravastatin $\geq 40 \mathrm{mg} \mathrm{QD}$, or any other statin in combination with ezetimibe.9 Systolic and diastolic blood pressure were recorded at the baseline visit. In addition, glycated hemoglobin Alc (HbAlc) and estimated glomerular filtration rate (eGFR) at baseline were also documented.

\section{Outcome Measures}

LDL-C concentration and other lipid concentrations, including HDL-C, total cholesterol (TC), and triglycerides, and, if feasible, apolipoprotein (Apo) A1, ApoB, ApoE, and lipoprotein(a) $(\mathrm{Lp}(\mathrm{a}))$, were measured as part of routine clinical practice at each clinical site at the baseline visit, 4 weeks (except Lp(a)), 12 weeks, or at the end of follow-up. LDL-C value was calculated using the Friedewald formula if it was not measured by direct assay. ${ }^{10}$ Non-HDL-C levels were calculated by subtracting HDL-C from the $\mathrm{TC}$ value.

AE (including information about seriousness and causal relationship to drug) and other safety information (e.g., overdose, lack of effectiveness, pregnancy, and lactation with or without AE) were collected. Adverse drug reactions (ADR) were defined as those AE for which the causal relationship with the drug could not be excluded. AE were coded using the Medical Dictionary for Regulatory Activities Japanese version 20.1.

\begin{tabular}{|c|c|c|c|c|}
\hline & HoFH & HeFH & $\mathrm{HC}$ & All patients \\
\hline $\mathrm{n}$ & 28 & 320 & 79 & 427 \\
\hline Sex, female & $13(46.4)$ & $127(39.7)$ & $24(30.4)$ & $164(38.4)$ \\
\hline \multicolumn{5}{|l|}{ Age } \\
\hline$<15$ years & $1(3.6)$ & $0(0.0)$ & $0(0.0)$ & $1(0.2)$ \\
\hline$\geq 15$ and $<65$ years & $21(75.0)$ & $168(52.5)$ & $30(38.0)$ & $219(51.3)$ \\
\hline$\geq 65$ years & $6(21.4)$ & $152(47.5)$ & $49(62.0)$ & 207 (48.5) \\
\hline$\geq 75$ years & $2(7.1)$ & $57(17.8)$ & $22(27.8)$ & $81(19.0)$ \\
\hline Age (years) & $51.9 \pm 16.4$ & $61.2 \pm 13.8$ & $66.9 \pm 10.3$ & $61.6 \pm 13.8$ \\
\hline $\mathrm{BMI}\left(\mathrm{kg} / \mathrm{m}^{2}\right)$ & $24.1 \pm 5.0$ & $25.2 \pm 4.1$ & $25.4 \pm 3.9$ & $25.2 \pm 4.1$ \\
\hline Outpatient & $26(92.9)$ & $302(94.4)$ & $67(84.8)$ & $395(92.5)$ \\
\hline \multicolumn{5}{|l|}{$\mathrm{FH}$ findings } \\
\hline Genetic test positive & $11(39.3)$ & $57(17.8)$ & $0(0.0)$ & $68(15.9)$ \\
\hline Tendon xanthoma & $21(75.0)$ & $162(50.6)$ & $3(3.8)$ & $186(43.6)$ \\
\hline Xanthoma tuberosum & $12(42.9)$ & $44(13.8)$ & $2(2.5)$ & $58(13.6)$ \\
\hline $\mathrm{CAD}$ & $22(78.6)$ & $246(76.9)$ & $62(78.5)$ & $330(77.3)$ \\
\hline DM & $5(17.9)$ & $89(27.8)$ & $48(60.8)$ & $142(33.3)$ \\
\hline IGT & $0(0.0)$ & $18(5.6)$ & $5(6.3)$ & $23(5.4)$ \\
\hline $\mathrm{HbA1c}(\%)^{\dagger}$ & $8.6 \pm 2.5(5)$ & $6.9 \pm 0.9(85)$ & $6.9 \pm 1.2(46)$ & $6.9 \pm 1.2(136)$ \\
\hline CKD & $4(14.3)$ & $32(10.0)$ & $22(27.8)$ & $58(13.6)$ \\
\hline Non-cardiogenic cerebral infarction & $1(3.6)$ & $22(6.9)$ & $11(13.9)$ & $34(8.0)$ \\
\hline
\end{tabular}




\begin{tabular}{|c|c|c|c|c|}
\hline & HoFH & $\mathrm{HeFH}$ & HC & All patients \\
\hline PAD & $4(14.3)$ & $42(13.1)$ & $12(15.2)$ & $58(13.6)$ \\
\hline \multicolumn{5}{|l|}{ Smoking } \\
\hline Absent & 19 (67.9) & $172(53.8)$ & 35 (44.3) & 226 (52.9) \\
\hline Current & $1(3.6)$ & $25(7.8)$ & $8(10.1)$ & $34(8.0)$ \\
\hline Past & $5(17.9)$ & $103(32.2)$ & $34(43.0)$ & $142(33.3)$ \\
\hline Hypertension & $12(42.9)$ & $200(62.5)$ & $66(83.5)$ & $278(65.1)$ \\
\hline $\mathrm{SBP}(\mathrm{mmHg})^{\ddagger}$ & $133.3 \pm 25.0(11)$ & $133.0 \pm 18.6(172)$ & $130.9 \pm 20.2(62)$ & $132.5 \pm 19.2(245)$ \\
\hline $\mathrm{DBP}(\mathrm{mmHg})^{\ddagger}$ & $70.3 \pm 10.8(11)$ & $74.3 \pm 12.4(172)$ & $73.2 \pm 12.5(62)$ & $73.8 \pm 12.4(245)$ \\
\hline Low HDL-C, $<40 \mathrm{mg} / \mathrm{dL}$ & $15(53.6)$ & $78(24.4)$ & $14(17.7)$ & $107(25.1)$ \\
\hline Family history of premature CAD & $6(21.4)$ & $133(41.6)$ & $7(8.9)$ & $146(34.2)$ \\
\hline \multicolumn{5}{|c|}{ Risk category (according to JAS guidelines) } \\
\hline Secondary prevention & $22(78.6)$ & $246(76.9)$ & $62(78.5)$ & $330(77.3)$ \\
\hline Primary prevention $\$$ & $6(21.4)$ & $72(22.5)$ & $16(20.3)$ & $94(22.0)$ \\
\hline High risk & NA & NA & $16(20.3)$ & NA \\
\hline Medium risk & NA & NA & 0 & NA \\
\hline Low risk & NA & NA & 0 & NA \\
\hline \multicolumn{5}{|l|}{ High-intensity statin medication" } \\
\hline Yes & $24(85.7)$ & $298(93.1)$ & $70(88.6)$ & $392(91.8)$ \\
\hline \multicolumn{5}{|l|}{ Combination therapy with statin } \\
\hline Yes & $26(92.9)$ & $313(97.8)$ & $76(96.2)$ & $415(97.2)$ \\
\hline Rosuvastatin & $15(53.6)$ & $179(55.9)$ & $31(39.2)$ & $225(52.7)$ \\
\hline Atorvastatin & $6(21.4)$ & $74(23.1)$ & $30(38.0)$ & $110(25.8)$ \\
\hline Pravastatin & $1(3.6)$ & $11(3.4)$ & $6(7.6)$ & $18(4.2)$ \\
\hline Pitavastatin & $4(14.3)$ & $56(17.5)$ & $11(13.9)$ & $71(16.6)$ \\
\hline Simvastatin & $0(0.0)$ & $1(0.3)$ & $1(1.3)$ & $2(0.5)$ \\
\hline Fluvastatin & $0(0.0)$ & $0(0.0)$ & $1(1.3)$ & $1(0.2)$ \\
\hline \multicolumn{5}{|c|}{ Combination therapy with other lipid medication } \\
\hline Yes & $21(75.0)$ & $210(65.6)$ & $27(34.2)$ & $258(60.4)$ \\
\hline Ezetimibe & $20(71.4)$ & $188(58.8)$ & $13(16.5)$ & $221(51.8)$ \\
\hline Probucol & $3(10.7)$ & $27(8.4)$ & $0(0.0)$ & $30(7.0)$ \\
\hline Colestimide & $4(14.3)$ & $39(12.2)$ & $2(2.5)$ & $45(10.5)$ \\
\hline Bezafibrate & $0(0.0)$ & $4(1.3)$ & $0(0.0)$ & $4(0.9)$ \\
\hline \multicolumn{5}{|l|}{ Prior medication } \\
\hline Antihypertensive & $15(53.6)$ & $206(64.4)$ & $58(73.4)$ & $279(65.3)$ \\
\hline Anti-diabetic & $2(7.1)$ & $55(17.2)$ & $28(35.4)$ & 85 (19.9) \\
\hline Antiplatelet & $16(57.1)$ & $222(69.4)$ & $58(73.4)$ & $296(69.3)$ \\
\hline Anticoagulant & $3(10.7)$ & $36(11.3)$ & $16(20.3)$ & $55(12.9)$ \\
\hline Anti-anginal & $13(46.4)$ & $178(55.6)$ & $47(59.5)$ & $238(55.7)$ \\
\hline \multicolumn{5}{|l|}{ Prior LDL-C apheresis } \\
\hline Yes & $14(50.0)$ & $25(7.8)$ & $0(0.0)$ & $39(9.1)$ \\
\hline Baseline LDL-C (mg/dL) & $228.8 \pm 157.9(28)$ & $152.6 \pm 44.3(320)$ & $113.9 \pm 36.1(79)$ & $150.4 \pm 62.8(427)$ \\
\hline Baseline TC (mg/dL) & $294.0 \pm 176.8(24)$ & $228.5 \pm 52.5(267)$ & $188.9 \pm 49.5(66)$ & $225.5 \pm 71.4(357)$ \\
\hline Baseline HDL-C (mg/dL) & $42.2 \pm 16.5(27)$ & $48.6 \pm 13.7(307)$ & $53.0 \pm 14.6(75)$ & $49.0 \pm 14.2(409)$ \\
\hline Baseline TG (mg/dL) & $127.2 \pm 88.1(27)$ & $141.5 \pm 80.6(312)$ & $160.5 \pm 112.6(76)$ & $144.1 \pm 88.0(415)$ \\
\hline Baseline non-HDL-C (mg/dL) & $251.4 \pm 176.9(24)$ & $180.0 \pm 48.8(254)$ & $135.0 \pm 46.7(64)$ & $176.6 \pm 70.8(342)$ \\
\hline \multicolumn{5}{|l|}{ eGFR $\left(\mathrm{mL} / \mathrm{min} / 1.73 \mathrm{~m}^{2}\right)$} \\
\hline$\geq 60$ & $20(71.4)$ & $189(59.1)$ & $40(50.6)$ & 249 (58.3) \\
\hline$\geq 45$ and $<60$ & $5(17.9)$ & $50(15.6)$ & $20(25.3)$ & $75(17.6)$ \\
\hline$\geq 30$ and $<45$ & $1(3.6)$ & $19(5.9)$ & $10(12.7)$ & $30(7.0)$ \\
\hline$\geq 15$ and $<30$ & $0(0.0)$ & $4(1.3)$ & $3(3.8)$ & $7(1.6)$ \\
\hline$<15$ & $0(0.0)$ & $0(0.0)$ & $1(1.3)$ & $1(0.2)$ \\
\hline
\end{tabular}

Data presented as $n(\%)$ for categorical variables and mean \pm SD or mean \pm SD $(n)$ for continuous variables. ${ }^{\dagger}$ Patients with DM or IGT. ‡Patients with hypertension. ${ }^{\$} \mathrm{HoFH}$ and $\mathrm{HeFH}$ were not subcategorized within primary prevention group. TJapan-specific definition: intensive if atorvastatin $\geq 10 \mathrm{mg} Q D$, rosuvastatin $\geq 5 \mathrm{mg} Q D$, simvastatin $\geq 20 \mathrm{mg} Q D$, fluvastatin $\geq 80 \mathrm{mg}$ QD, pitavastatin $\geq 2 \mathrm{mg}$ QD, pravastatin $\geq 40 \mathrm{mg}$ $\mathrm{QD}$, or any statin use in combination with ezetimibe. The name of the statin, its daily dose, and treatment start and end dates were collected on the CRF. BMI, body mass index; CAD, coronary artery disease; CKD, chronic kidney disease; CRF, case report form; DBP, diastolic blood pressure; DM, diabetes mellitus; eGFR, estimated glomerular filtration rate; FH, familial hypercholesterolemia; HbA1c, glycated hemoglobin; $\mathrm{HC}$, hypercholesterolemia; HDL-C, high-density lipoprotein cholesterol; $\mathrm{HeFH}$, heterozygous familial hypercholesterolemia; $\mathrm{HoFH}$, homozygous familial hypercholesterolemia; IGT, impaired glucose tolerance; JAS, Japan Atherosclerosis Society; LDL-C, low-density lipoprotein cholesterol; NA, not applicable; PAD, peripheral artery disease; QD, once daily; SBP, systolic blood pressure; TC, total cholesterol; TG, triglycerides. 


\begin{tabular}{|c|c|c|c|c|}
\hline & \multicolumn{3}{|c|}{ Effectiveness analysis set I } & HC \\
\hline Baseline LDL-C (mg/dL) & Mean \pm SD & $204.7 \pm 88.7$ & $152.1 \pm 44.3$ & $112.3 \pm 34.8$ \\
\hline \multirow{4}{*}{$\begin{array}{l}\% \text { change from baseline to } \\
12 \text { weeks }\end{array}$} & Mean \pm SD & $-45.5 \pm 27.0$ & $-54.2 \pm 29.0$ & $-64.6 \pm 22.4$ \\
\hline & $95 \% \mathrm{Cl}$ & -57.2 to -33.8 & -57.6 to -50.8 & -69.8 to -59.3 \\
\hline & P-value ${ }^{\dagger}$ & $<0.001$ & $<0.001$ & $<0.001$ \\
\hline & $\mathrm{n}^{\ddagger}$ & 23 & 280 & 72 \\
\hline \multicolumn{5}{|l|}{ Effectiveness analysis set II } \\
\hline Baseline LDL-C (mg/dL) & Mean \pm SD & $201.7 \pm 74.0$ & $151.1 \pm 42.8$ & $107.5 \pm 29.8$ \\
\hline \multirow{4}{*}{$\begin{array}{l}\% \text { change from baseline to } \\
12 \text { weeks }\end{array}$} & Mean \pm SD & $-49.3 \pm 16.5$ & $-61.0 \pm 22.1$ & $-65.5 \pm 20.9$ \\
\hline & $95 \% \mathrm{Cl}$ & -59.8 to -38.8 & -64.1 to -57.9 & -70.9 to -60.1 \\
\hline & P-value ${ }^{\dagger}$ & $<0.001$ & $<0.001$ & $<0.001$ \\
\hline & $\mathrm{n}^{\ddagger}$ & 12 & 198 & 59 \\
\hline
\end{tabular}

†One-sample t-test. fPatients with data available for \%change from baseline to week 12. Abbreviations as in Table 1.

\section{Statistical Analysis}

Summary statistics for continuous variables included the number of patients, mean, median, and SD. For categorical variables, the frequency and percentage were calculated. Missing data were not imputed. The statistical difference between lipid levels at baseline and at 12 weeks was determined using the 1-sample t-test. All reported P-values are 2-sided based on the 1-sample t-test, and 2-sided 95\% CI were calculated. Multiplicity adjustments were not considered; therefore, all P-values were nominal, and no formal hypothesis testing was conducted.

The safety analysis set included all patients who had received at least 1 dose of evolocumab, had data available for safety assessment, and did not meet any prespecified criteria. The effectiveness analysis set I consisted of those patients from the safety analysis set who did not use evolocumab off label, were enrolled within the enrollment period, had evaluable efficacy data, were not lost to followup after the first visit, and in whom LDL-C data were adequate and calculated using the correct method. The effectiveness analysis set II included those patients from the effectiveness analysis set $\mathrm{I}$ in whom evolocumab dosage and use were according to the package insert, and the daily dose of concomitant statin did not exceed the prescribed dose at the time of initiation of this drug; who were $>15$ years; and who were co-prescribed statin at the time of evolocumab initiation.

The target sample size for the safety analysis set was determined as 3,000 patients, with an observation period of 2 years for $95 \%$ statistical power to detect at least 1 patient experiencing an $\mathrm{AE}$ with a 2-year incidence of $0.1 \%$. A sample size of 6,000 enrolled patients over the 4-year enrollment period was chosen to achieve the aforementioned target for 2-year follow-up data, with an assumed discontinuation rate (lost to follow-up) of $50 \%$, which was estimated based on PMS for Hydroxymethylglutaryl coenzyme A (HMG-CoA) reductase inhibitors in Japan. The additional factors that were considered while estimating the sample size included the difference in the mode of administration of evolocumab and the anticipated challenges in monitoring patients who were transferred from specialized care hospitals to general practice clinics. This report provided safety and effectiveness based on the data collected until 17 January 2018. Statistical analysis was conducted by A2 Healthcare Corporation (Tokyo) and funded by the study sponsors. Statistical analysis was conducted using SAS, version 9.2 (SAS Institute, Cary, NC, USA).

\section{Results}

\section{Patient Disposition}

A total of 427 patients from 299 clinical sites were included in the safety analysis set after excluding 1 patient due to protocol violation. Of the patients included in the safety analysis set, 398 were eligible for inclusion in the effectiveness analysis set I, and 313 for the effectiveness analysis set II. Patient disposition, including reasons for exclusion from each analysis set, is presented in Figure 1.

\section{Patient Characteristics}

The safety analysis set consisted of $28 \mathrm{HoFH}, 320 \mathrm{HeFH}$, and 79 HC patients. Mean age was 61.6 years, and $38.4 \%$ of patients were female (Table 1). The median follow-up duration was 85.0 days. Of the safety analysis population, patients with major risk factors consisted of 77.3\% (76.9$78.6 \%$ by diagnosis) with a medical history of CAD; $38.6 \%$ (67.1\% of HC patients) with that of DM or IGT with mean HbA1c $6.9 \%$; and $65.1 \%$ (83.5\% of HC patients) with that of hypertension. Patients with a history of other risk factors consisted of $13.6 \%$ with CKD, $8.0 \%$ with non-cardiogenic cerebral infarction, $13.6 \%$ with PAD, 25.1\% with low HDL-C ( $<40 \mathrm{mg} / \mathrm{dL}), 34.2 \%$ with a family history of premature CAD, and $8.0 \%$ who were habitual current smokers. Overall, $77.3 \%$ of patients were in the secondary prevention and $22.0 \%$ in the primary prevention risk category, according to JAS guidelines; ${ }^{6}$ all 16 HC patients for primary prevention were categorized as having high risk. High-intensity statin was used in $91.8 \%$ of patients (85.7-93.1\% by diagnosis). LDL apheresis had been conducted in $50 \%$ of $\mathrm{HoFH}$ and in $7.8 \%$ of $\mathrm{HeFH}$ patients. At baseline, mean LDL-C was $150.4 \mathrm{mg} / \mathrm{dL}$ in the overall population, and $228.8 \mathrm{mg} / \mathrm{dL}$ in the $\mathrm{HoFH}$ patients. Mean HDL-C at baseline was $49.0 \mathrm{mg} / \mathrm{dL}$ and the non-HDL-C was $176.6 \mathrm{mg} / \mathrm{dL}$. Statin was co-prescribed in $97.2 \%$ of patients, and ezetimibe in $51.8 \%$. Other frequently used medications were antihypertensives $(65.3 \%)$, antiplatelets (69.3\%), anti-anginals (55.7\%), and anti-diabetics (19.9\%). 


\begin{tabular}{|c|c|c|c|}
\hline \multirow[b]{3}{*}{ TC } & \multicolumn{3}{|c|}{ \%change from baseline to 12 weeks } \\
\hline & HoFH & HeFH & $\mathrm{HC}$ \\
\hline & & & \\
\hline Mean \pm SD & $-32.2 \pm 26.1$ & $-38.0 \pm 16.6$ & $-40.3 \pm 15.8$ \\
\hline $95 \% \mathrm{Cl}$ & -44.8 to -19.7 & -40.2 to -35.8 & -44.4 to -36.2 \\
\hline P-value $^{\dagger}$ & $<0.001$ & $<0.001$ & $<0.001$ \\
\hline $\mathrm{n}^{\ddagger}$ & 19 & 222 & 60 \\
\hline \multicolumn{4}{|l|}{ HDL-C } \\
\hline Mean \pm SD & $11.0 \pm 21.6$ & $10.0 \pm 19.9$ & $8.8 \pm 17.1$ \\
\hline $95 \% \mathrm{Cl}$ & 1.7 to 20.4 & 7.7 to 12.4 & 4.6 to 13.0 \\
\hline P-value ${ }^{\dagger}$ & 0.023 & $<0.001$ & $<0.001$ \\
\hline $\mathrm{n}^{\ddagger}$ & 23 & 268 & 67 \\
\hline \multicolumn{4}{|l|}{ TG } \\
\hline Mean \pm SD & $-2.8 \pm 63.0$ & $-3.2 \pm 44.3$ & $-13.8 \pm 32.8$ \\
\hline $95 \% \mathrm{Cl}$ & -30.0 to 24.5 & -8.5 to 2.1 & -21.7 to -5.8 \\
\hline $\mathrm{P}_{\text {-value }}{ }^{\dagger}$ & 0.835 & 0.239 & $<0.001$ \\
\hline $\mathrm{n}^{\ddagger}$ & 23 & 271 & 68 \\
\hline \multicolumn{4}{|l|}{ Non-HDL-C } \\
\hline Mean \pm SD & $-40.5 \pm 30.0$ & $-50.1 \pm 21.2$ & $-59.8 \pm 19.6$ \\
\hline $95 \% \mathrm{Cl}$ & -55.0 to -26.1 & -53.0 to -47.2 & -65.0 to -54.7 \\
\hline P-value $^{\dagger}$ & $<0.001$ & $<0.001$ & $<0.001$ \\
\hline $\mathrm{n}^{\ddagger}$ & 19 & 212 & 58 \\
\hline
\end{tabular}

†One-sample t-test. :Patients with data available for \%change from baseline to week 12 for the respective lipid parameter. Abbreviations as in Table 1.

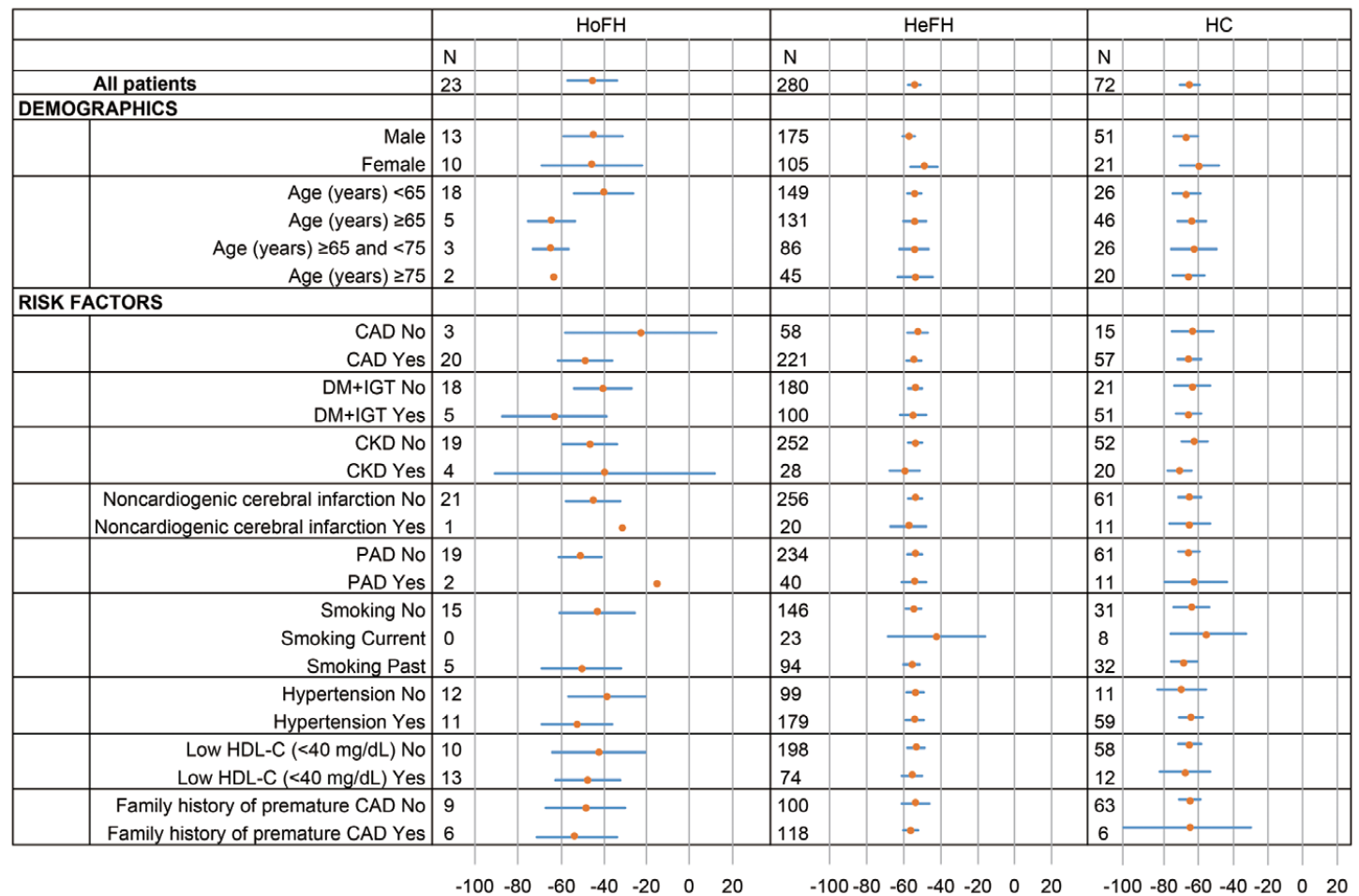

LDL-C Percent Change From Baseline to 12 weeks (\%)

Figure 2. Subgroup analysis of percent change from baseline in low-density lipoprotein cholesterol (LDL-C) at 12 weeks, according to homozygous familial hypercholesterolemia $(\mathrm{HoFH})$, heterozygous familial hypercholesterolemia $(\mathrm{HeFH})$, and hypercholesterolemia $(\mathrm{HC}$; effectiveness analysis set I). Orange dots, mean; blue bars, $95 \% \mathrm{Cl}$. If the sample size is $<3,95 \% \mathrm{Cl}$ is not shown. CAD, coronary artery disease; CKD, chronic kidney disease; DM, diabetes mellitus; HDL-C, high-density lipoprotein cholesterol; IGT, impaired glucose tolerance; PAD, peripheral artery disease. 
LDL-C Percent Change From Baseline by HoFH

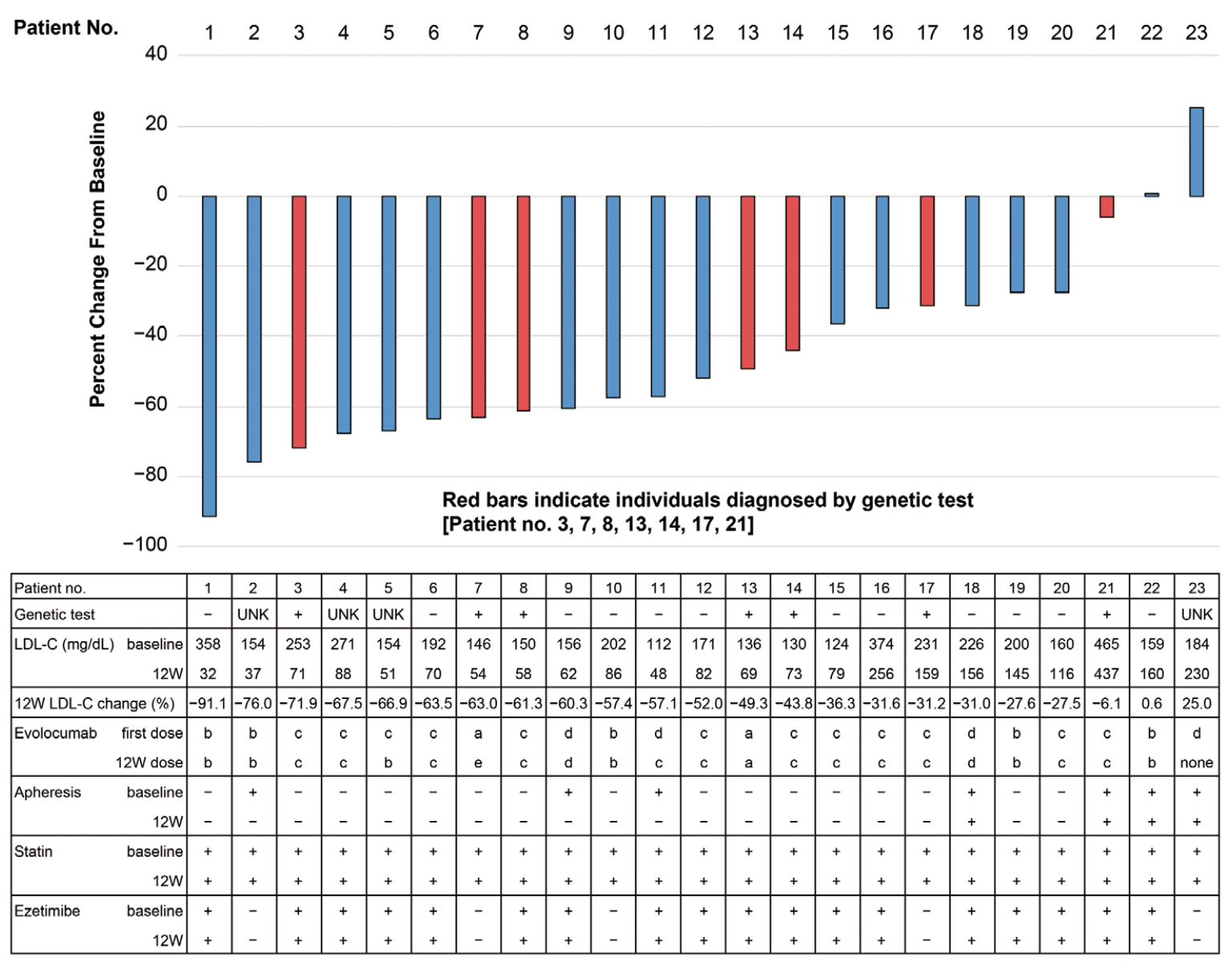

Figure 3. Waterfall plot of percent change in low-density lipoprotein cholesterol (LDL-C) at 12 weeks in patients with homozygous familial hypercholesterolemia (HoFH). Genetic test: -, no; +, yes. Evolocumab dose: a, $280 \mathrm{mg} / 4$ weeks; b, $140 \mathrm{mg} / 2$ weeks; c, $420 \mathrm{mg} / 4$ weeks or 5 weeks; d, 420 mg/2 weeks; e, $280 \mathrm{mg} / 5$ weeks or 6 weeks. UNK, unknown; W, week.

\begin{tabular}{|c|c|c|c|c|c|}
\hline & \multirow{2}{*}{$\begin{array}{l}\text { All patients } \\
n=427\end{array}$} & \multicolumn{4}{|c|}{ Lowest achieved LDL-C category } \\
\hline & & $\begin{array}{c}\geq 40 \mathrm{mg} / \mathrm{dL} \\
\mathrm{n}=236^{\dagger}\end{array}$ & $\begin{array}{c}<40 \mathrm{mg} / \mathrm{dL} \\
\mathrm{n}=187^{\dagger}\end{array}$ & $\begin{array}{c}\geq 25 \text { and }<40 \mathrm{mg} / \mathrm{dL} \\
\mathrm{n}=107^{\dagger}\end{array}$ & $\begin{array}{c}<25 \mathrm{mg} / \mathrm{dL} \\
\mathrm{n}=80^{\dagger}\end{array}$ \\
\hline $\mathrm{AE}$ & $56(13.1)$ & $38(16.1)$ & $18(9.6)$ & $11(10.3)$ & $7(8.8)$ \\
\hline $\mathrm{ADR}^{\ddagger}$ & $23(5.4)$ & $16(6.8)$ & $7(3.7)$ & $5(4.7)$ & $2(2.5)$ \\
\hline
\end{tabular}

Data given as $n(\%)$. †Patients with LDL-C data. $\ddagger^{A} A$ reported by the site physicians. ADR, adverse drug reaction; $A E$, adverse event; LDL-C, low-density lipoprotein cholesterol.

\section{Effectiveness}

In the primary endpoint analysis of effectiveness, the percent change from baseline in mean \pm SD LDL-C was $-45.5 \% \pm$ $27.0 \%$ in $\mathrm{HoFH}(\mathrm{P}<0.001$ vs. baseline [the same applies hereafter] $),-54.2 \% \pm 29.0 \%$ in $\mathrm{HeFH}(\mathrm{P}<0.001)$, and $-64.6 \% \pm 22.4 \%$ in $\mathrm{HC}(\mathrm{P}<0.001)$ patients after 12 weeks of evolocumab treatment. All percent changes in mean LDL-C at 12 weeks were statistically significant when compared with baseline values (Table 2). In the sensitivity analyses of the effectiveness analysis set II, the percent change from baseline in mean $\pm \mathrm{SD}$ LDL-C was $-49.3 \% \pm 16.5 \%$ in
HoFH $(\mathrm{P}<0.001),-61.0 \% \pm 22.1 \%$ in $\mathrm{HeFH}(\mathrm{P}<0.001)$, and $-65.5 \% \pm 20.9 \%$ in $\mathrm{HC}(\mathrm{P}<0.001)$ patients. As for other lipid parameters, favorable changes at 12 weeks for TC, HDL-C, and non-HDL-C were observed across diagnosis (Table 3). Changes in ApoA1, ApoB, ApoE, and Lp(a) at 12 weeks were statistically significant (Supplementary Table 1). The percentage of patients with $\mathrm{HoFH}, \mathrm{HeFH}$, and $\mathrm{HC}$ who achieved the LDL-C goal for comprehensive risk management was $34.8 \%, 65.7 \%$, and $97.2 \%$, respectively (Supplementary Table 2).

On subgroup analyses of sex, age, and CV risk factors 
(CAD, DM and IGT, CKD, non-cardiogenic cerebral infarction, PAD, smoking, hypertension, low HDL-C $[<40 \mathrm{mg} / \mathrm{dL}]$, and family history of premature CAD), the 12-week LDL-C-lowering effectiveness of evolocumab did not differ between subgroups (Figure 2). A waterfall plot for individual HoFH patients showed a reduction in LDL-C value after 12 weeks of evolocumab treatment in combination with statin, ezetimibe, or apheresis as expected, although some patients had a weaker response to evolocumab treatment (Figure 3).

Overall, the incidence rate of $\mathrm{AE}$ was $13.1 \%$ (56/427), and that of ADR was $5.4 \%$ (23/427). AE were most frequently reported in the system organ classes (SOC) "cardiac disorders" $(2.6 \%)$ and "investigations" (2.1\%). Most ADR were reported in the SOC "investigations", "general disorders and administration site conditions", and "gastrointestinal disorders". ADR by preferred term are presented in Supplementary Table 3. Subgroup analysis by lowest achieved LDL-C after evolocumab treatment suggested that the incidence of $\mathrm{AE}$ and $\mathrm{ADR}$ was not associated with the lowest achieved LDL-C values (Table 4).

\section{Discussion}

There are very few reports on the effectiveness and safety of evolocumab treatment in a large number of patients with $\mathrm{FH}$, especially in those with $\mathrm{HoFH}$, in the real-world setting. This is the first study to present results of evolocumab from a large sample population, and the results show that LDL-C lowering by the anti-PCSK 9 antibody evolocumab was beneficial in patients with $\mathrm{FH}(\mathrm{HoFH}$ and $\mathrm{HeFH}$ ) as well as $\mathrm{HC}$ when assessed at 12 weeks in the real-world setting.

The Japanese patients in this study were characterized by a relatively high risk in several aspects. First, FH patients with both homozygous and heterozygous genotypes represented a large majority of the enrolled population. Second, patients with $\mathrm{HC}$ with pre-existing $\mathrm{CAD}$ were predominant. In a randomized, controlled clinical trial conducted in Japan, HC patients with pre-existing CAD comprised approximately $25 \%$ of the clinical study population; ${ }^{9}$ in contrast, the proportion was more than 3 -fold $(78.5 \%)$ in the current study.

The frequency of FH in Asian countries, including Japan, has been estimated to be 1 in 500 people. ${ }^{11}$ Recently updated guidelines, however, focus on, and increase awareness about, diagnosis and management goals for $\mathrm{FH}$ patients, ${ }^{6}$ thereby, improving diagnostic rates and management. The availability of a new LDL-C-lowering therapy in the form of a PCSK9 inhibitor could be particularly beneficial for FH patients and could be a reason for the high proportion of FH patients with CAD in this real-world study. The baseline LDL-C value, however, was relatively high in FH patients despite combination therapy with high-intensity statins. This study showed that evolocumab in combination with high-intensity statins was prescribed in the real-world setting to patients diagnosed with $\mathrm{FH}$ and primarily to those with $\mathrm{HC}$, who were in the high $\mathrm{CV}$ risk group. Thus, as shown in this study so far, it is important to assess the relevant risk factors of each patient individually prior to initiating evolocumab treatment.

In the primary endpoint analysis of the effectiveness analysis set I, after 12 weeks of evolocumab treatment, mean \pm SD LDL-C percent changes were $-45.5 \% \pm 27.0 \%$, $-54.2 \% \pm 29.0 \%$, and $-64.6 \% \pm 22.4 \%$ in $\mathrm{HoFH}, \mathrm{HeFH}$, and
HC patients, respectively. This study population, however, included patients who did not follow the dosage and administration of evolocumab specified in package insert, ${ }^{4}$ including that for lower doses and dose frequency. The effectiveness analysis set II that included patients who followed the package insert guidelines for dosage was used to summarize the percent change from baseline in LDL-C levels to investigate the impact of adherence to package insert guidelines on the effectiveness of evolocumab. The mean \pm SD LDL-C percent changes in the effectiveness analysis set II were $-49.3 \% \pm 16.5 \%,-61.0 \% \pm 22.1 \%$, and $-65.5 \% \pm 20.9 \%$ in $\mathrm{HoFH}, \mathrm{HeFH}$, and $\mathrm{HC}$ patients, respectively, thus suggesting that evolocumab is more effective with small variations in its efficacy in patients with $\mathrm{HoFH}$, $\mathrm{HeFH}$, and $\mathrm{HC}$, when prescribed as per the package insert guidelines. ${ }^{4}$ The change at 12 weeks in other lipid parameters and ApoA1, ApoB, and Lp(a) with evolocumab treatment showed a trend similar to that observed in the clinical trial. Compared with the response to evolocumab treatment observed for $\mathrm{HC}$ or $\mathrm{HeFH}$ patients, the waterfall plot for those with HoFH showed that not all patients responded to evolocumab treatment. Although LDL-C lowering is achievable with the anti-PCSK9 monoclonal antibody in $\mathrm{HoFH}$ patients, no reduction was reported in those patients who were receptor negative in a previous study. 12

On subgroup effectiveness analysis, the profile of LDL-C percent change at 12 weeks was similar in each subgroup. The degree of LDL-C lowering in this study was similar to that observed in previous clinical trials in HC.,13 The waterfall plot for HoFH patients suggested that the LDLC-lowering effectiveness of evolocumab at 12 weeks in the real-world setting was not affected by the genetic test conducted for the diagnosis of $\mathrm{HoFH}$.

The incidence of ADR was $5.4 \%$. Stratification by minimum achieved LDL-C after evolocumab treatment suggested that the incidence of reported AE and ADR was not associated with the lowest achieved LDL-C values. No association between evolocumab treatment or very low LDL-C and the emergence of AE has been reported in long-term controlled clinical trials. ${ }^{5}$

Although long-term, randomized, controlled clinical trials have been conducted or are ongoing, this observational study in the real-world setting provides useful information for clinical practice because the real-world setting allowed for inclusion of a broader range of HC patients, without the limitation of exclusion criteria as in controlled clinical trials. ${ }^{14,15}$ Furthermore, a longer evolocumab exposure and observation period enabled greater insights into the LDLC-lowering effect and safety profile of evolocumab in the real-world setting.

\section{Study Limitations}

We recognize several potential limitations of this study. First, $\mathrm{HoFH}$ patients included in this study could be $\mathrm{HeFH}$ patients because diagnosis was not carried out according to the prescribed procedure of genetic testing; some of the patients diagnosed on genetic testing, however, had an LDL-C-lowering profile as observed in the waterfall plot. Second, because of the reduced number of FH patients (especially $\mathrm{HoFH}$ ), there was limited power to provide meaningful results in the subgroup analyses. Finally, the effectiveness results from this study cannot be compared with that of clinical trials because of the single-arm observational design of this study. Also, unlike in a blinded, 
randomized clinical study that is conducted under strictly controlled conditions, good adherence to and persistence of evolocumab in combination with statin treatment may not be possible in the real-world setting; patients in this observational study, however, may be representative of those in routine clinical practice.

\section{Conclusions}

This real-world study demonstrated that patients on evolocumab treatment are primarily those with $\mathrm{FH}$ and $\mathrm{HC}$ in the secondary prevention group. These results add to the current pool of knowledge about LDL-C-lowering therapy with evolocumab for $\mathrm{FH}$, both $\mathrm{HoFH}$ and $\mathrm{HeFH}$, and could be useful for physicians when choosing an appropriate option for medical treatment.

\section{Acknowledgments}

A2 Healthcare Corporation (Tokyo, Japan) performed statistical analysis, which was funded by Amgen Astellas BioPharma and Astellas Pharma. Cactus Communications (Mumbai, India) provided editorial and medical writing support, which was funded by Amgen Astellas BioPharma and Astellas Pharma.

\section{Data Sharing}

Qualified researchers may request data from Amgen clinical studies. Complete details are available at https://wwwext.amgen.com/science/ clinical-trials/clinical-data-transparency-practices/

\section{Author Contributions}

All authors made substantial contributions to the concept and design, or analysis and interpretation of data, and to the drafting of the manuscript, or revising it critically for important intellectual content. All authors provided final approval of the manuscript.

\section{Ethics Approval}

The protocol of this observational study (evolocumab post-marketing survey) was approved by the Pharmaceuticals and Medical Devices Agency of Japan prior to the study. The study was conducted in accordance with the applicable Pharmaceuticals and Medical Devices law, Good Post-Marketing Study Practice of Japan and the Declaration of Helsinki.

\section{Informed Consent}

Written informed consent was obtained from all participating patients.

\section{Disclosures}

K.Y. has received remuneration (e.g., lecture fees) from Kowa Pharmaceutical, MSD, Astellas Pharma, Amgen Astellas BioPharma, Takeda Pharmaceutical, Sanofi, AstraZeneca, and Pfizer Japan, and research funding from Takeda Pharmaceutical, MSD, Pfizer Japan, and Teijin Pharma Limited. J.A. has received remuneration (e.g., lecture fees) from Amgen Astellas BioPharma. K.K. has received remuneration (e.g., lecture fees) from Bayer, Boehringer Ingelheim, Daiichi-Sankyo, and Kyowa-Hakko Kirin, and research funding from Sanofi, Boehringer Ingelheim, Bayer, Daiichi-Sankyo, KyowaHakko Kirin, and Takeda Pharmaceutical. H.I. was an employee of Amgen Astellas BioPharma and holds stock/stock options of Amgen. K.A. is an employee of Amgen Astellas BioPharma and holds stock/ stock options of Amgen. T.S. was an employee of Amgen Astellas BioPharma. Y.S. and J.S. are employees of Amgen Astellas BioPharma. T.T. has received remuneration (e.g., lecture fees) from Sanofi, MSD, Bayer Yakuhin, Astellas, and scholarship funds/donations from Takeda Pharmaceutical.

\section{Funding}

This study was sponsored by Amgen Astellas BioPharma K.K. and Astellas Pharma Inc.

\section{References}

1. Law MR, Wald NJ, Rudnicka AR. Quantifying effect of statins on low density lipoprotein cholesterol, ischaemic heart disease, and stroke: Systematic review and meta-analysis. BMJ 2003; 326: 1423.

2. Teramoto T, Uno K, Miyoshi I, Khan I, Gorcyca K, Sanchez $\mathrm{RJ}$, et al. Low-density lipoprotein cholesterol levels and lipidmodifying therapy prescription patterns in the real world: An analysis of more than 33,000 high cardiovascular risk patients in Japan. Atherosclerosis 2016; 252: 248-254.

3. Santos RD, Gidding SS, Hegele RA, Cuchel MA, Barter RJ, Watts GF, et al. Defining severe familial hypercholesterolemia and the implications for clinical management: A consensus statement from the International Atherosclerosis Society Severe Familial Hypercholesterolemia Panel. Lancet Diabetes Endocrinol 2016; 4: $850-861$.

4. Pharmaceuticals and Medical Devices Agency, Japan. [Repatha ${ }^{\circledR}$ Package Insert.] (in Japanese) https://www.pmda.go.jp/ PmdaSearch/iyakuDetail/ResultDataSetPDF/112922_2189401G 1020_1_04 (accessed December 12, 2018).

5. Sabatine MS, Giugliano RP, Keech AC, Honarpour N, Wiviott SD, Murphy SA, et al. Evolocumab and clinical outcomes in patients with cardiovascular disease. $N$ Engl J Med 2017; 376: $1713-1722$.

6. Kinoshita M, Yokote K, Arai H, Iida M, Ishigaki Y, Ishibashi $\mathrm{S}$, et al. Committee for Epidemiology and Clinical Management of Atherosclerosis. Japan Atherosclerosis Society (JAS) Guidelines for Prevention of Atherosclerotic Cardiovascular Diseases 2017. J Atheroscler Thromb 2018; 25: 846-984.

7. Pharmaceuticals and Medical Devices Agency, Japan. [Repatha ${ }^{\circledR}$ Risk management plan.] (in Japanese) http://www.pmda.go.jp/ RMP/www/112922/85027e01-9772-4ddb-b68c-f9409d0fc962/112 922_2189401G1020_001RMP.pdf (accessed December 17, 2018).

8. Teramoto T, Sasaki J, Ishibashi S, Birou S, Daida H, Dohi S, et al. Executive summary of the Japan Atherosclerosis Society (JAS) guidelines for the diagnosis and prevention of atherosclerotic cardiovascular diseases in Japan - 2012 version. J Atheroscler Thromb 2013; 20: 517-523.

9. Hirayama A, Honarpour N, Yoshida M, Yamashita S, Huang F, Wasserman SM, et al. Effects of evolocumab (AMG 145), a monoclonal antibody to PCSK9, in hypercholesterolemic, statintreated Japanese patients at high cardiovascular risk: Primary results from the phase 2 YUKAWA study. Circ J 2014; 78: 1073-1082.

10. Friedewald WT, Levy RI, Fredrickson DS. Estimation of the concentration of low-density lipoprotein cholesterol in plasma, without use of the preparative ultracentrifuge. Clin Chem 1972; 18: $499-502$.

11. Zhou M, Zhao D. Familial hypercholesterolemia in Asian populations. J Atheroscler Thromb 2016; 23: 539-549.

12. Stein EA, Honarpour N, Wasserman SM, Xu F, Scott, R, Raal FJ. Effect of the proprotein convertase subtilisin/kexin 9 monoclonal antibody, AMG 145, in homozygous familial hypercholesterolemia. Circulation 2013; 128: 2113-2120.

13. Kiyosue A, Honarpour N, Kurtz C, Xue A, Wasserman SM, Hirayama A. A phase 3 study of evolocumab (AMG 145) in statin-treated Japanese patients at high cardiovascular risk. Am J Cardiol 2016; 117: 40-47.

14. Black N. Why we need observational studies to evaluate the effectiveness of health care. BMJ 1996; 312: 1215-1218.

15. Hirayama A, Yamashita S, Inomata H, Kassahun H, Cyrille M, Ruzza A, et al. One-year efficacy and safety of evolocumab in Japanese patients: A pooled analysis from the open-label extension OSLER studies. Circ J 2017; 81: 1029-1035.

\section{Supplementary Files}

Please find supplementary file(s);

http://dx.doi.org/10.1253/circrep.CR-19-0027 